\title{
e-13. Epilepsy in Association with Intracranial Tumor
}

\author{
Kazuo Mori, Shiro Waga, Tsunemaro Koyama, Kaoru Iwayama, \\ Yasuhiro Yonekawa, Hiroyuki Mizuno, Nobuo Fushikı and \\ Hajime HANDA \\ Department of Neurosurgery, Kyoto Universtiy, School of Medicine
}

\begin{abstract}
Statistical analyses were made in 161 cases of intracranial tumor with epileptic seizures in our series of 1942-1968.

In 95 cases of these 161 , a seizure manifested as an initial disorder.

In the glioma group affecting the frontal or temporal lobe, development of other symptoms and signs was delayed for a long time. This was particularly common in tumors of a frontal location: in 10 cases out of these 22, seizures remained as an isolated symptom for more than 3 years.

There was no difference between a duration in which seizures were isolated and various different histological types of glioma.

In the infratentorial tumor, no cases had seizures as an initial disorder. In these cases, seizures appeared after tumor was so widely spread as to lead to a rise in intracranial pressure.

In 14 cases of the falx or parasagittal meningioma in which a seizure manifested as an initial symptom, 11 cases showed focal Jacksonian type. In 7 cases of these 14, a possible loss of motor power came out within 3 months after the manifestation of the first attack. On the contrary, in about half of those affecting the convexity, seizures were non-focal and remained as an isolated symptom for more than 1 year.

The present report emphasizes that careful observation is necessary even in patients with seizures which manifested in their ten or twenties.
\end{abstract}

\section{e-14. Experimental Study on Acute Increased Intracranial Pressure (Symptoms and Signs associated with Increased Infratentorial Pressure)}

\author{
Toshiaki Machida, Tamotsu Takeda and Minoru Tsunoda \\ Dept. of Neurosurgery, Sapporo Municipal Hospital
}

Takeshi Kashiwaba and Hiroshi ABE

Dept. of Neurosurgery, Hokkaido University 